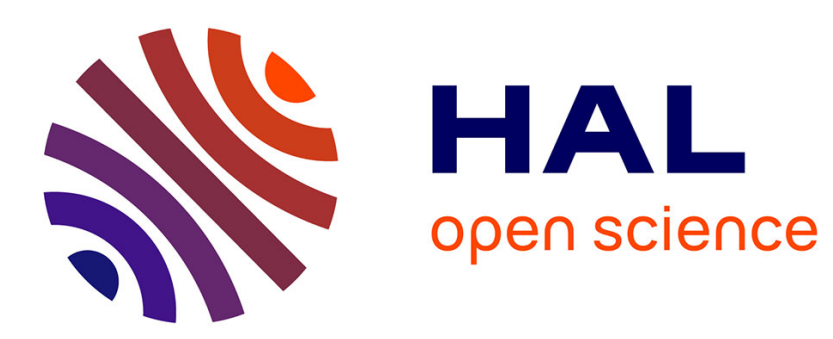

\title{
Les adoptions en France et en Italie : une histoire comparée du droit et des pratiques (XIXe-XXIe siècles)
}

Jean-François Mignot

\section{To cite this version:}

Jean-François Mignot. Les adoptions en France et en Italie: une histoire comparée du droit et des pratiques (XIXe-XXIe siècles). Population (édition française), 2015, 70 (4), pp.805-830. 10.3917/popu.1504.0805 . halshs-01326723

\section{HAL Id: halshs-01326723 \\ https://shs.hal.science/halshs-01326723}

Submitted on 5 Jun 2016

HAL is a multi-disciplinary open access archive for the deposit and dissemination of scientific research documents, whether they are published or not. The documents may come from teaching and research institutions in France or abroad, or from public or private research centers.
L'archive ouverte pluridisciplinaire HAL, est destinée au dépôt et à la diffusion de documents scientifiques de niveau recherche, publiés ou non, émanant des établissements d'enseignement et de recherche français ou étrangers, des laboratoires publics ou privés. 


\section{Les adoptions en France et en Italie : une histoire comparée du droit et des pratiques $\left(\mathrm{XIX}^{\mathrm{e}}-\mathrm{XXI}^{\mathrm{e}}\right.$ siècles)}

\section{Table des matières}

\section{Introduction}

1. Des histoires du droit de l'adoption fortement similaires

a. Une adoption simple de majeurs à visée successorale, en France (1804-1923) et en

Italie (1865-1942)

b. Une adoption simple à visée non plus seulement successorale mais aussi de protection de l'enfance, en France (1923-1939) et en Italie (1942-1967)

c. Une adoption plénière, en France (depuis 1939) et en Italie (depuis 1967)................... 4

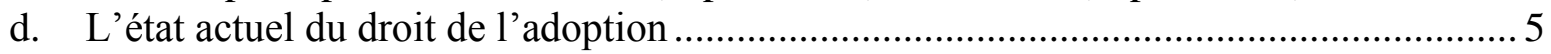

2. Le nombre d'adoptés simples et pléniers ..............................................................9

3. Le nombre et le profil des adoptés pléniers nationaux et internationaux .................. 12

a. Le faible nombre de mineurs nationaux adoptables plénièrement ................................ 12

b. Le développement puis la récente baisse des adoptions internationales ........................ 13

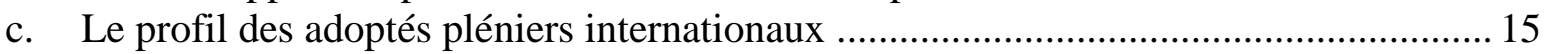

Conclusion.................................................................................................................................................... 17

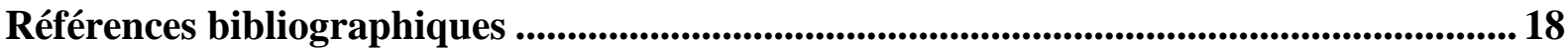

\section{Résumé de 200 à 230 mots}

La France et l'Italie connaissent des droits de l'adoption proches et font partie, depuis les années 1990, des pays qui adoptent le plus de mineurs à l'international. Mais sur plus long terme, dans quelle mesure les pratiques de l'adoption sont-elles similaires entre ces deux pays ? Pour le savoir, cet article retrace l'histoire du droit et des pratiques de l'adoption en France et en Italie depuis le XIX ${ }^{\mathrm{e}}$ siècle. Il apparaît que si la France et l'Italie ont connu des histoires du droit de l'adoption très proches, leurs nombres actuels d'adoptions simples sont très différents, principalement en raison du fait que les recompositions familiales après divorce sont beaucoup plus rares en Italie qu'en France. En revanche, les nombres d'adoptions plénières et les profils des adoptés pléniers sont similaires dans les deux pays : après avoir été confrontés au faible nombre de mineurs nationaux adoptables, les candidats à l'adoption deux pays sont confrontés, depuis 2011 surtout, à la baisse du nombre de mineurs adoptables à l'international. En conséquence, les adoptés internationaux en France et en Italie tendent à être plus âgés, plus souvent en fratrie, et plus souvent malades ou handicapés qu'auparavant.

\section{Mots-clés}

Adoption ; filiation ; divorce ; comparaison internationale ; démographie historique ; France ; Italie. 


\section{Introduction}

La France et l'Italie connaissent aujourd'hui des droits de l'adoption qui sont relativement similaires et qui se distinguent, notamment, du droit de l'adoption des pays anglo-saxons. En outre, depuis au moins les années 1990, la France et l'Italie font partie des pays qui, avec l'Espagne et derrière les États-Unis, adoptent le plus grand nombre de mineurs à l'international. Parmi les pays développés, la France et l'Italie se distinguent ainsi d'autres pays comme l'Allemagne ou a fortiori le Royaume-Uni, qui n'adoptent que peu à l'international, sans parler du Japon qui, pour ainsi dire, n'adopte pas de mineurs étrangers. Mais sur plus long terme, dans quelle mesure les pratiques de l'adoption sont-elles similaires entre la France et l'Italie ? Et que révèlent les différences entre ces deux pays ? Pour le savoir, le présent article propose une histoire comparée de l'adoption en France et en Italie depuis le $\mathrm{XIX}^{\mathrm{e}}$ siècle.

L'adoption est une institution juridique qui crée un lien de filiation entre un adoptant et un adopté, sans que ce lien de filiation repose sur la procréation de l'adopté par l'individu ou le couple adoptant. L'adoption permet ainsi à l'adopté d'hériter du nom de famille et du patrimoine de l'adoptant. ${ }^{1}$ On distingue aussi deux types d'adoption: «plénière » et « simple ». L'adoption plénière crée, entre un mineur adopté et un couple ou un individu adoptant, un lien de filiation qui remplace le lien de filiation entre l'adopté et ses parents d'origine. L'adoption plénière crée ainsi un lien nouveau, substitutif et exclusif entre l'adopté et l'adoptant, en conséquence de quoi l'adopté hérite son nom et ses droits de succession de sa seule famille adoptive. Par contraste, l'adoption simple ne crée entre l'adopté, souvent majeur, et l'adoptant qu'un lien de filiation qui s'ajoute au lien de filiation entre l'adopté et ses parents d'origine. L'adoption simple conduit ainsi l'adopté à ajouter à son nom et à ses droits de succession d'origine ceux qu'il tire de son adoptant.

Si l'adoption intéresse de longue date l'anthropologie (Fine 1998), la sociologie (Fisher 2003) et la psychologie (Savard, 2010), ce n'est que depuis récemment que les démographes ont commencé à analyser ce phénomène. Aujourd'hui encore, l'étude de l'adoption n'a qu'une place marginale en démographie, comme en témoigne sa quasi-absence dans le manuel de référence de la discipline (Caselli et al., 2001). En effet, l'impact de l'adoption sur la dynamique des populations est négligeable, et l'adoption ne peut s'étudier qu'en lien avec le droit, une discipline qui a peu de contacts avec la démographie. Toutefois, l'essor de l'adoption internationale a fait de l'adoption une modalité de la migration (Weil, 1984). En outre, depuis les années 1940, l'adoption internationale est liée à des crises démographiques (guerre, maladie ou famine) dans les pays émetteurs, même si ces pays ne sont pas les plus pauvres ou les plus féconds de la planète, et l'adoption internationale est aussi liée à diverses évolutions démographiques et juridiques dans les pays d'accueil, où la libéralisation de la contraception et de l'interruption volontaire de grossesse a réduit le nombre de mineurs abandonnés et donc adoptables (Selman, 2002).

Toujours d'un point de vue démographique, il est particulièrement intéressant de comparer les pratiques d'adoption entre la France et l'Italie, et ce pour au moins deux raisons. D'une part, aucun pays occidental autre que l'Italie n'est doté, depuis aussi longtemps, d'un droit de l'adoption similaire au droit français. Par conséquent, comparer les pratiques d'adoption entre ces deux pays permet de repérer d'éventuelles variations «à environnement juridique contrôlé », c'est-à-dire des différences de comportements qui ne sont pas dues à des différences entre les droits de l'adoption mais à des différences d'opportunités ou de désir

\footnotetext{
${ }^{1}$ L'adoption se distingue de certaines institutions apparentées, notamment « l'affiliation » (affiliazione), qui a existé en droit italien de 1939 à 1983. L'affiliation transférait la puissance paternelle à l'affiliant, et elle permettait à l'affilié de bénéficier de droits alimentaires pendant sa minorité et même de prendre le nom de l'affiliant. Toutefois, l'affiliation ne créait pas de lien de filiation : l'affilié ne succédait pas à l'affiliant. Le nombre d'affiliations en Italie a chuté, de 1955 à 1979, de près de 2500 à moins de 500 par an (Brand, 1985).
} 
d'adopter, c'est-à-dire des différences de comportements démographiques. D'autre part, la comparaison entre les pratiques d'adoption en France et en Italie permet d'aborder non seulement les adoptions plénières (notamment internationales), mais aussi les adoptions simples, qui n'existent pas dans les pays de common law comme les États-Unis - pays sur lequel, du reste, n'existent pas de données de long terme sur les adoptions, que ces données soient agrégées ou a fortiori individuelles (Carp, 1998 ; Lovelock, 2000 ; Herman, 2008 ; Davis, 2011).

Cet article présente une histoire comparée du droit et des pratiques de l'adoption en France et en Italie depuis le $\mathrm{XIX}^{\mathrm{e}}$ siècle. Nous passons tout d'abord en revue l'histoire du droit de l'adoption depuis que cette institution a été introduite en France (1804) et en Italie (1865). Nous abordons ensuite l'évolution du nombre annuel d'adoptés simples et pléniers. Enfin, parmi les adoptés pléniers, nous distinguons les adoptés nationaux des adoptés internationaux, en cherchant à dégager leurs effectifs et leurs profils, ainsi que les évolutions démographiques qu'ils révèlent.

\section{Des histoires du droit de l'adoption fortement similaires}

Le droit de l'adoption détermine qui peut adopter, qui peut être adopté, quelle est la procédure à suivre pour adopter et enfin quels sont les effets juridiques de l'adoption. Pour analyser son évolution depuis le $\mathrm{XIX}^{\mathrm{e}}$ siècle, nous distinguons globalement trois étapes successives: tout d'abord, n'existe en droit qu'une adoption simple de majeurs, à visée successorale; puis existe aussi une adoption simple de mineurs, à visée de protection de l'enfance ; enfin, est introduite une adoption plénière. Ce mouvement général s'observe aussi bien en France (Gutton, 1993 ; Neirinck, 2000 ; Halifax, 2007 ; Murat 2008) qu'en Italie (Brand, 1985 ; Center for Adoption Policy, 2014 ; Sénat, 2014), même si l'Italie ne suit ce mouvement qu'avec un décalage temporel par rapport à la France.

a. Une adoption simple de majeurs à visée successorale, en France (18041923) et en Italie (1865-1942)

L'adoption est introduite dans les droits français et italien en même temps que les premiers codes civils du XIX ${ }^{\mathrm{e}}$ siècle, eux-mêmes fortement inspirés du droit romain, qui connaît une adoption de majeur capable (sui juris) à visée successorale, appelée « adrogation ». En France, le Code civil de 1804 introduit une adoption de majeurs de 21 ans. Peuvent adopter les hommes et les femmes de plus de cinquante ans et de tout statut matrimonial, pourvu qu'ils soient sans enfant légitime. L'adoption a deux effets majeurs : elle ajoute au nom de l'adopté celui de l'adoptant, et elle confère à l'adopté les mêmes droits sur la succession de l'adoptant que ceux d'un enfant légitime. L'adoption ne rompt donc pas les liens qu'a l'adopté avec sa famille d'origine : elle ne crée qu'une filiation additive. En outre, l'adoption crée pour l'adopté plusieurs prohibitions à mariage: dans sa famille adoptive, l'adopté ne peut se marier ni avec son adoptant (ou son conjoint), ni avec les autres enfants (biologiques ou adoptifs) du même adoptant. Enfin, l'adoption crée entre l'adoptant et l'adopté des obligations alimentaires réciproques. Toutefois, l'adoption ne fait pas entrer l'adopté dans la famille de l'adoptant, en ce sens que l'adopté n'hérite que de l'adoptant, et non pas des ascendants ni des collatéraux de l'adoptant.

De même, en Italie, le Code civil de 1865, qui est le premier code civil de l'Italie unifiée et qui est très fortement inspiré du Code Napoléon, introduit une adoption de majeurs de 18 ans (même si l'âge de la majorité civile est de 21 ans). Précisément, ne peuvent être adoptés que les majeurs dont les parents consentent à l'adoption. Peuvent adopter les hommes et les femmes de plus de cinquante ans et de tout statut matrimonial, pourvu qu'ils soient sans enfant légitime ou légitimé et pourvu qu'ils aient au moins dix-huit ans de plus que l'adopté. Concernant les effets de l'adoption, ils sont absolument identiques à ceux du Code civil français, à ceci près qu'une personne ne peut pas adopter son enfant naturel (alors qu'en 
France, tout au long du XIX ${ }^{\mathrm{e}}$ siècle, la plupart des adoptions sont le fait de personnes adoptant leur enfant naturel afin qu'il puisse hériter comme le ferait un enfant légitime). Comme en France, l'adoption crée une filiation additive : elle ne rompt pas les liens de l'adopté avec sa famille d'origine et ne fait qu'ajouter au nom et aux droits de succession de l'adopté ceux qu'il tire de l'adoptant.

b. Une adoption simple à visée non plus seulement successorale mais aussi de protection de l'enfance, en France (1923-1939) et en Italie (1942-1967)

La Première Guerre mondiale provoque environ 1400000 morts en France et 650000 en Italie (Rohrbasser, 2014). Dans ce contexte, l'adoption pouvait être conçue comme l'un des modes de prise en charge des orphelins de guerre non recueillis par leur famille étendue (Jablonka, 2006). Ainsi en France, la loi du 19 juin 1923 rend adoptables les mineurs. Peuvent adopter un mineur les majeurs de quarante ans sans enfant légitime, comme c'est le cas des couples inféconds mais aussi des nombreux couples qui ont perdu leur seul enfant pendant la guerre. L'adoption de mineurs confère à l'adoptant la «puissance paternelle » sur l'adopté, c'est-à-dire l'ensemble de droits et de devoirs que le père avait à l'égard de ses enfants mineurs (et qui a été remplacée, depuis la loi du 4 juin 1970, par «l'autorité parentale »). Toutefois, la loi n'autorise toujours qu'une adoption simple, qui crée une filiation additive.

En Italie, le décret royal $n^{\circ} 1357$ du 31 juillet 1919 et la loi $n^{\circ} 1458$ du 24 septembre 1940, tous deux relatifs à l'adoption des orphelins de guerre, témoignent du fait que le législateur commence à concevoir l'adoption comme un outil d'aide sociale à l'enfance abandonnée. Cela dit, ce n'est qu'en 1942 que le nouveau Code civil rend adoptables les mineurs. Peuvent adopter un mineur les majeurs de cinquante ans sans enfant légitime (Brand, 1985). Comme en France, l'adoption de mineurs confère à l'adoptant la puissance paternelle, mais la loi n'autorise toujours qu'une adoption simple. Après-guerre, les dispositions du Code civil de 1942 relatives à l'adoption sont expurgées de leurs éléments d'inspiration raciste, notamment l'interdiction d'adopter des non-aryens (décret royal du 20 janvier 1944).

c. Une adoption plénière, en France (depuis 1939) et en Italie (depuis 1967)

En France, le décret-loi du 29 juillet 1939, aussi appelé Code de la famille, introduit en droit l'adoption plénière («légitimation adoptive » de 1939 à 1966, «adoption plénière » depuis 1966). Ce nouveau type d'adoption fait non seulement entrer l'enfant adopté dans la famille de l'adoptant mais en outre — c'est là le point crucial — rompt les liens, notamment successoraux, de l'adopté avec sa famille d'origine. En conséquence, ce nouveau type d'adoption garantit l'exclusivité du lien entre les parents adoptifs et leur enfant et apaise donc certaines réticences à adopter, en plus de garantir à l'adopté qu'il ne sera pas déplacé d'une famille à une autre. En adoption plénière, peuvent adopter les couples qui sont mariés depuis une certaine durée (dix ans en 1939, deux ans depuis 1996), dont au moins un membre remplit une condition d'âge (trente-cinq ans en 1941, vingt-huit depuis 1996) et un certain écart d'âge avec l'adopté (au moins quinze ans de plus que l'adopté depuis 1966), et qui (jusqu'en 1976) n'ont pas d'enfant légitime (depuis 1976, peuvent aussi adopter les couples ou les individus qui ont déjà des enfants, légitimes ou non). En outre, depuis 1966, peuvent adopter plénièrement non plus seulement des époux, mais des individus agissant seuls, sous certaines conditions d'âge (trente ans en 1966, vingt-huit ans depuis 1996). En adoption plénière, peut être adopté un mineur d'un certain âge (cinq ans en 1939, quinze ans depuis 1966) qui est orphelin ou abandonné. En adoption simple, peuvent depuis 1966 adopter les mêmes couples et les mêmes personnes qui peuvent adopter en adoption plénière; et peut être adoptée une personne sans condition d'âge. Même si les dispositions de 1939 sont réorganisées par la loi du 11 juillet 1966, c'est donc fondamentalement depuis 1939 que coexistent en France deux types d'adoption: une adoption simple, qui crée une filiation additive, et une adoption plénière, qui crée une filiation substitutive. 
En Italie, c'est la loi n431 du 5 juin 1967 qui introduit en droit l'adoption plénière. Comme en France, ce nouveau type d'adoption, destiné à donner une famille aux mineurs abandonnés, fait entrer l'adopté dans la famille de l'adoptant mais surtout rompt les liens de filiation de l'adopté avec sa famille d'origine. De 1967 jusqu'en 1983, l'adoption plénière est appelée «adoption spéciale» (adozione speciale), pour la distinguer de l'adoption simple, appelée «adoption ordinaire» (adozione ordinaria). En adoption plénière, peuvent alors adopter les époux qui ont au moins trente-cinq ans, qui sont mariés depuis au moins cinq ans et qui ne sont pas séparés ; et peut être adopté un mineur, pourvu qu'il ait au moins dix-huit ans de moins que l'adoptant. En adoption simple, peuvent adopter les personnes ou les couples sans enfant, l'objectif étant pour elles de transmettre leur nom et leur patrimoine à un mineur ou à un majeur adoptif, lorsque les deux parties sont consentantes. Ces dispositions sont réorganisées par la loi $\mathrm{n}^{\circ} 184$ du 4 mai 1983 : l'adoption simple, désormais réservée aux majeurs de 18 ans, s'appelle « adoption de majeurs » (adozione di persone maggiori di età), et l'adoption plénière, désormais réservée aux mineurs (abandonnés ou autres), s'appelle «adoption de mineurs » (adozione di minori). En outre, depuis la loi n 149 du 28 mars 2001, l'écart d'âge maximal entre l'adoptant et le mineur adopté plénier est passé de 40 à 45 ans. Mais c'est bien depuis 1967 que coexistent en Italie les deux grands types d'adoption, simple et plénière.

\section{d. L'état actuel du droit de l'adoption}

Actuellement, les droits français et italien de l'adoption sont très proches. D'une part, ces deux droits distinguent une adoption simple, à visée principalement successorale, d'une adoption plénière, à visée éducative et dont le principal objectif, pour les pouvoirs publics, est de promouvoir l'intérêt de l'enfant adopté. Au fil du temps, on a observé en France et en Italie la même évolution: la modalité privilégiée de l'adoption devient l'adoption plénière, et non plus l'adoption simple; en outre, pour le législateur, l'adoption plénière ne vise plus véritablement à satisfaire l'intérêt de l'adoptant (élever un enfant) ni a fortiori celui des parents d'origine de l'adopté (les dispenser d'élever leur enfant), mais à satisfaire l'intérêt de l'adopté lui-même (lui donner des parents aimants). D'autre part, dans les deux pays les dispositions du droit de l'adoption plénière sont similaires (Tableau 1, qui ne concerne que l'adoption extrafamiliale, l'adoption intrafamiliale étant soumise à moins de conditions). En effet la France et l'Italie ont ratifié la Convention de La Haye sur la protection des enfants et la coopération en matière d'adoption internationale, et elles ont modifié leurs droits de l'adoption internationale voire nationale en conséquence. La Convention est entrée en vigueur en France en 1998 et en Italie en 2000 (Conférence de La Haye de droit international privé, 2014). Cela dit, une différence importante subsiste entre les droits français et italien de l'adoption plénière. En France, les hommes et les femmes agissant seuls, notamment les personnes homosexuelles, peuvent depuis 1966 adopter plénièrement un mineur (même si l'homosexualité déclarée a souvent motivé des refus d'agrément), et les couples mariés de personnes de même sexe peuvent eux aussi, depuis la loi du 17 mai 2013, adopter plénièrement un mineur. Par contraste, en Italie les personnes agissant seules ne peuvent adopter plénièrement, et en l'absence de mariage de personnes de même sexe les couples homosexuels ne peuvent généralement pas adopter plénièrement. Si l'analyse démographique de l'adoption par les couples de personnes de même sexe reste à faire en France, tel n'est pas le cas aux Etats-Unis, où des travaux proprement démographiques portent sur ce sujet (Davis, 2013).

Notons enfin que les droits français et italien sont aussi relativement proches en matière d'accès des enfants abandonnés et adoptés à leurs origines : si cet accès reste le plus souvent difficile, il s'est récemment libéralisé. La France et l'Italie sont deux des rares pays du monde dont le Code civil autorise une mère à accoucher de façon anonyme et à ne pas reconnaître son enfant (Lefaucheur, 2006). Concrètement, une mère peut ne pas déclarer son nom sur 
l'acte de naissance de l'enfant dont elle a accouché. En conséquence, les enfants nés dans le secret («sous $X »)$ ont longtemps eu le plus grand mal, en France comme en Italie, à connaître des informations sur leurs géniteurs, que ces informations soient non identifiantes (état de santé des géniteurs, circonstances et raisons qui ont mené à l'abandon, etc.) ou a fortiori identifiantes (nom et prénom des géniteurs et de leurs autres enfants). Le plus souvent, les adoptés nationaux qui ne sont pas nés dans le secret, notamment les quelques adoptés qui ont été reconnus avant d'être abandonnés ou d'être consentis à l'adoption, ont moins de difficultés à connaître leurs origines.

En Italie, c'est la loi nº 149 du 28 mars 2001 qui libéralise l'accès des adoptés à leurs origines : désormais, à l'âge de 25 ans (la majorité civile est, depuis 1975, de 18 ans), l'adopté peut s'adresser au tribunal des mineurs pour avoir accès à des informations, identifiantes ou non, sur ses géniteurs. Cela dit, si l'adopté est né dans le secret ou si au moins l'un de ses géniteurs s'est opposé à la révélation de son identité, il ne lui est pas permis de connaître l'identité de ses géniteurs. En France, c'est depuis la loi du 22 janvier 2002 relative à l'accès aux origines des personnes adoptées et pupilles de l'État que le secret de la filiation est réversible : l'adopté majeur peut s'adresser au Conseil national pour l'accès aux origines personnelles (CNAOP) pour avoir accès à des informations sur ses géniteurs (pour une approche quantitative de l'activité du CNAOP, voir Voisin et Georges, 2011). Mais là encore, si les géniteurs de l'adopté né dans le secret refusent de lever le secret de leur identité, l'adopté n'est pas autorisé à connaître leur identité. Ni en Italie ni en France, donc, n'est reconnu un droit général des enfants nés sous $\mathrm{X}$ (même devenus adultes) à connaître leurs origines ni a fortiori l'identité de leurs géniteurs. En Italie toutefois, la Cour constitutionnelle a établi en 2013 le droit de l'enfant à connaître ses origines même si la mère a demandé le maintien de son anonymat, si cela est essentiel pour garantir la santé de l'enfant. 
Tableau 1. Le droit de l'adoption en France et en Italie en 2014

\begin{tabular}{|c|c|c|c|c|c|}
\hline & & \multicolumn{2}{|c|}{ France } & \multicolumn{2}{|c|}{ Italie } \\
\hline & & Adoption simple & Adoption plénière & $\begin{array}{l}\text { Adoption simple (adozione } \\
\text { di persone maggiori d'età) }\end{array}$ & $\begin{array}{l}\text { Adoption plénière } \\
\text { (adozione dei minori) }\end{array}$ \\
\hline \multirow{4}{*}{$\begin{array}{l}\text { Les } \\
\text { conditions } \\
\text { que } \\
\text { doivent } \\
\text { remplir les } \\
\text { adoptés }\end{array}$} & $\begin{array}{l}\text { Âge et statut } \\
\text { matrimonial }\end{array}$ & $\begin{array}{l}\text { Avoir au moins } 15 \text { ans de moins } \\
\text { que l'adoptant }\end{array}$ & $\begin{array}{c}\text { Avoir moins de } 15 \text { ans, et avoir au } \\
\text { moins } 15 \text { ans de moins que } \\
\text { l'adoptant }\end{array}$ & $\begin{array}{l}\text { Avoir plus de } 18 \text { ans, pas déjà } \\
\text { adopté, avoir au moins } 18 \text { ans de } \\
\text { moins que l'adoptant et ne pas être } \\
\text { son enfant né hors mariage }\end{array}$ & $\begin{array}{c}\text { Avoir moins de } 18 \text { ans, avoir entre } \\
18 \text { et } 45 \text { ans de moins que } \\
\text { l'adoptant (exceptions permises) et } \\
\text { ne pas être son enfant né hors } \\
\text { mariage }\end{array}$ \\
\hline & $\begin{array}{l}\text { Perte de lien } \\
\text { de filiation }\end{array}$ & - & $\begin{array}{c}\text { Être sans famille («pupille de } \\
\text { l'État » ou déclaré abandonné par } \\
\text { jugement du tribunal), ou avoir été } \\
\text { expressément consenti à } \\
\text { l'adoption par ses parents }\end{array}$ & - & $\begin{array}{l}\text { Être sans famille et avoir été } \\
\text { déclaré adoptable par le tribunal } \\
\text { (dichiarazione di adottabilità) }\end{array}$ \\
\hline & Consentement & $\begin{array}{l}\text { Consentement nécessaire si } \\
\text { l'adopté a plus de } 15 \text { ans }\end{array}$ & $\begin{array}{l}\text { Consentement nécessaire si } \\
\text { l'adopté a plus de } 13 \text { ans }\end{array}$ & Consentement nécessaire & $\begin{array}{l}\text { Consentement nécessaire si } \\
\text { l'adopté a plus de } 14 \text { ans }\end{array}$ \\
\hline & $\begin{array}{c}\text { Durée de } \\
\text { placement } \\
\text { dans la future } \\
\text { famille } \\
\end{array}$ & & Au moins 6 mois & & $\begin{array}{l}\text { Au moins } 1 \text { an (affidamento } \\
\text { preadottivo) }\end{array}$ \\
\hline \multirow{2}{*}{$\begin{array}{l}\text { Les } \\
\text { conditions } \\
\text { que } \\
\text { doivent } \\
\text { remplir les } \\
\text { adoptants }\end{array}$} & $\begin{array}{l}\text { Âge et statut } \\
\text { matrimonial }\end{array}$ & \multicolumn{2}{|c|}{$\begin{array}{c}\text { Avoir plus de } 28 \text { ans, ou être un couple marié depuis plus de } 2 \text { ans ou } \\
\text { dont les deux membres ont plus de } 28 \text { ans }\end{array}$} & $\begin{array}{c}\text { Avoir au moins } 35 \text { ans (sauf } \\
\text { exceptions) et être sans enfant } \\
\text { légitime ou légitimé }\end{array}$ & $\begin{array}{l}\text { Être des époux majeurs de } 18 \text { ans, } \\
\text { mariés depuis au moins } 3 \text { ans } \\
\text { (durée de cohabitation avant } \\
\text { mariage incluse), non séparés et } \\
\text { capables d'élever des mineurs }\end{array}$ \\
\hline & Agrément & & $\begin{array}{l}\text { Nécessaire, il est accordé par le } \\
\text { service départemental de l'Aide } \\
\text { sociale à l'enfance pour cinq ans }\end{array}$ & & $\begin{array}{l}\text { Nécessaire, il est accordé par le } \\
\text { tribunal des mineurs pour trois ans }\end{array}$ \\
\hline \multirow{2}{*}{$\begin{array}{l}\text { La } \\
\text { procédure } \\
\text { d'adoption }\end{array}$} & Administrative & & $\begin{array}{l}\text { L'Aide sociale à l'enfance, qui } \\
\text { recueille les mineurs adoptables, } \\
\text { apparente le mineur adoptable et } \\
\text { son adoptant }\end{array}$ & & \\
\hline & Judiciaire & $\begin{array}{c}\text { Le jugement d'adoption est } \\
\text { prononcé par un tribunal (tribunal } \\
\text { de grande instance) }\end{array}$ & $\begin{array}{l}\text { Le jugement d'adoption est } \\
\text { prononcé par un tribunal (tribunal } \\
\text { de grande instance) ou, s'il est } \\
\text { prononcé par un pays étranger } \\
\text { reconnaissant l'adoption plénière, } \\
\text { il n'a pas à être prononcé en }\end{array}$ & $\begin{array}{l}\text { Le jugement d'adoption est } \\
\text { prononcé par un tribunal } \\
\text { (tribunale civile) }\end{array}$ & $\begin{array}{l}\text { Le jugement d'adoption } \\
\text { (dichiarazione di adozione) est } \\
\text { prononcé par un tribunal } \\
\text { (tribunale per i minorenni) }\end{array}$ \\
\hline
\end{tabular}




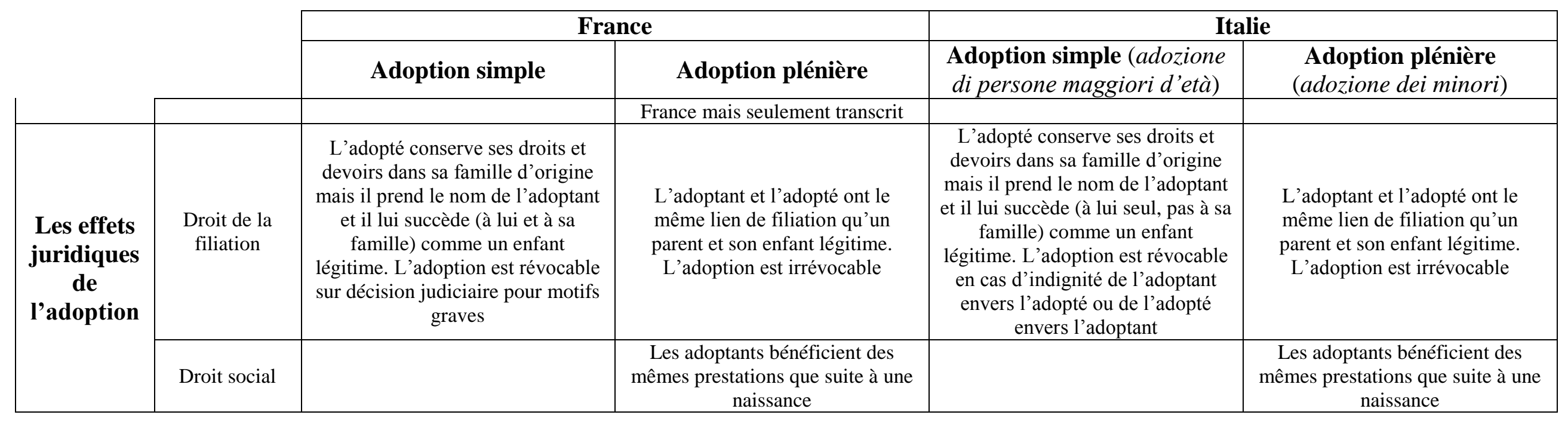




\section{Le nombre d'adoptés simples et pléniers}

Depuis que l'adoption existe dans les droits français et italien, comment les pratiques d'adoption ont-elles évolué ? Pour le savoir, nous commençons par comparer les nombres d'adoptions entre les deux pays.

\section{Encadré 1. Les sources statistiques sur l'adoption en France et en Italie}

La France et l'Italie sont deux des rares pays dans lesquels existent des données statistiques de long terme aussi bien sur les adoptions simples que sur les adoptions plénières. Dans ces deux pays une administration centralisée recense chaque année les adoptions.

En France, la principale source d'information est une publication du Ministère de la Justice qui a connu plusieurs intitulés et que nous appelons ici Compte général (Ministère de la Justice, 1841-1932, 1933-1960, 1961-1976, 1980). Il s'agit d'un annuaire qui recense les jugements d'adoption simple depuis 1841 (données indisponibles de 1804 à 1840) et les jugements d'adoption plénière depuis 1952 (données indisponibles de 1939 à 1951). Cette publication ne comportant plus d'informations sur l'adoption depuis le début des années 1980, elle est complétée par deux enquêtes ponctuelles que le Ministère de la Justice a réalisées, respectivement, sur les années 1992 (Belmokhtar, 1996) et 2007 (Belmokhtar, 2009). Ces annuaires et ces enquêtes ponctuelles renseignent sur les adoptions simples et plénières, qu'elles soient nationales ou internationales, mais parmi les jugements d'adoption plénière d'enfants étrangers elles ne recensent que les jugements prononcés en France (et non ceux prononcés à l'étranger). Par ailleurs, en ce qui concerne les adoptions internationales (pour la quasi-totalité d'entre elles, des adoptions plénières), le Ministère des Affaires étrangères rapporte, depuis 1979, le nombre de visas «adoption » qu'il délivre à des mineurs au cours de chaque année (Ministère des Affaires étrangères, 2014). Comme le jugement d'adoption d'un enfant ramené en France ne peut être prononcé qu'au moins six mois après son arrivée, et qu'un tribunal peut refuser la demande d'adoption, le nombre de visas «adoption » délivrés une année ne renseigne pas rigoureusement sur le nombre d'adoptés internationaux au cours de la même année. Cela dit, l'évolution du nombre de visas «adoption » délivrés au fil des ans donne une bonne idée des tendances temporelles. Enfin, concernant les adoptions nationales de pupilles de l'État, l'Observatoire national de l'enfance en danger recense le nombre de mineurs nationaux adoptables (et qui, lorsqu'ils sont adoptés, font presque exclusivement l'objet d'adoptions plénières) (Observatoire national de l'enfance en danger, 2007-2013).

En Italie, les principales sources d'information sont les publications de divers Ministères ou autres institutions (Ministero di agricoltura, industria e commercio de 1891 à 1906, Ministero di grazia e giustizia de 1907 à 1935, Istat, Movimento dei procedimenti civili de 1936 à 1996, Ministero della giustizia depuis 1997). En 2011, à l'occasion des 150 ans de l'unification de l'Italie, l'Institut national de statistique Istat a rassemblé et publié ces séries historiques, qui recensent les jugements d'adoption simple à partir de 1891 (données indisponibles de 1865 à 1890) et jusqu'en 1983 (données indisponibles depuis 1984) ainsi que les jugements d'adoption plénière depuis 1968 (Istat, 2011). En outre, en ce qui concerne les adoptions internationales, plusieurs institutions rapportent, depuis les années 1990, le nombre de mineurs internationaux autorisés à entrer sur le territoire italien (Istituto degli innocenti, 2008 ; Commissione per le adozioni internazionali, 2014). Comme en France, le nombre de mineurs autorisés à entrer sur le territoire ne renseigne pas rigoureusement sur le nombre d'adoptés internationaux en Italie au cours de la même année, mais il donne une bonne idée des tendances temporelles.

Notons enfin que, malheureusement, aucune source ne renseigne sur l'évolution du 
profil des femmes ou des couples nationaux ou internationaux qui ont confié leur enfant à l'adoption. Il s'agit là de l'aspect le plus méconnu de l'histoire de l'adoption, que ce soit en France ou en Italie mais aussi dans la plupart des autres pays (sur le cas de l'Ukraine voir Mykytyn-Gazziero, 2014).

Les nombres d'adoptés simples en France et en Italie ont connu deux trajectoires parallèles du XIX ${ }^{\mathrm{e}}$ siècle jusqu'au milieu des années 1970 (Figure 1). En France, tant que l'adoption est une adoption simple de majeurs à visée successorale (1804-1923), on compte environ une centaine d'adoptions simples par an. En Italie, alors que l'adoption est là aussi une adoption simple de majeurs à visée successorale (1865-1942), on compte une centaine d'adoptions simples par an jusqu'à la Première Guerre, puis de 200 à 500 adoptions simples par an entre-deux-guerres, et plus d'un millier d'adoptions simples par an au tout début de la Seconde Guerre. Puis dans les deux pays l'introduction d'une adoption simple de mineurs à visée non plus seulement successorale mais aussi de protection de l'enfance contribue à augmenter fortement le nombre d'adoptions simples par an. En France, de 1923 à 1939, le nombre d'adoptions simples par an dépasse le millier, sans qu'on connaisse malheureusement la part des adoptés simples qui sont des mineurs. En Italie, de 1942 à 1967, le nombre d'adoptions simples par an dépasse les 2000 , la plupart de ces adoptés étant des mineurs (entre 1955 et 1979, de $55 \%$ à $80 \%$ des adoptions simples portent sur des mineurs (Brand, 1985)). Enfin, l'introduction dans les deux pays d'une adoption plénière (1939 en France, 1967 en Italie) tend, jusqu'au milieu des années 1970, à réduire le nombre d'adoptés simples : dans bien des cas, les adoptants préfèrent s'attacher l'adopté par un lien substitutif et donc exclusif, plutôt qu'additif (Marmier, 1969). En France de l'après-guerre (en partie marqué par les effets du conflit mondial) jusqu'au milieu des années 1970, et plus encore en Italie à partir de 1967, le nombre annuel d'adoptions simples tend à décliner.

Figure 1. Nombre annuel d'adoptés simples en France et en Italie, 1841-2007

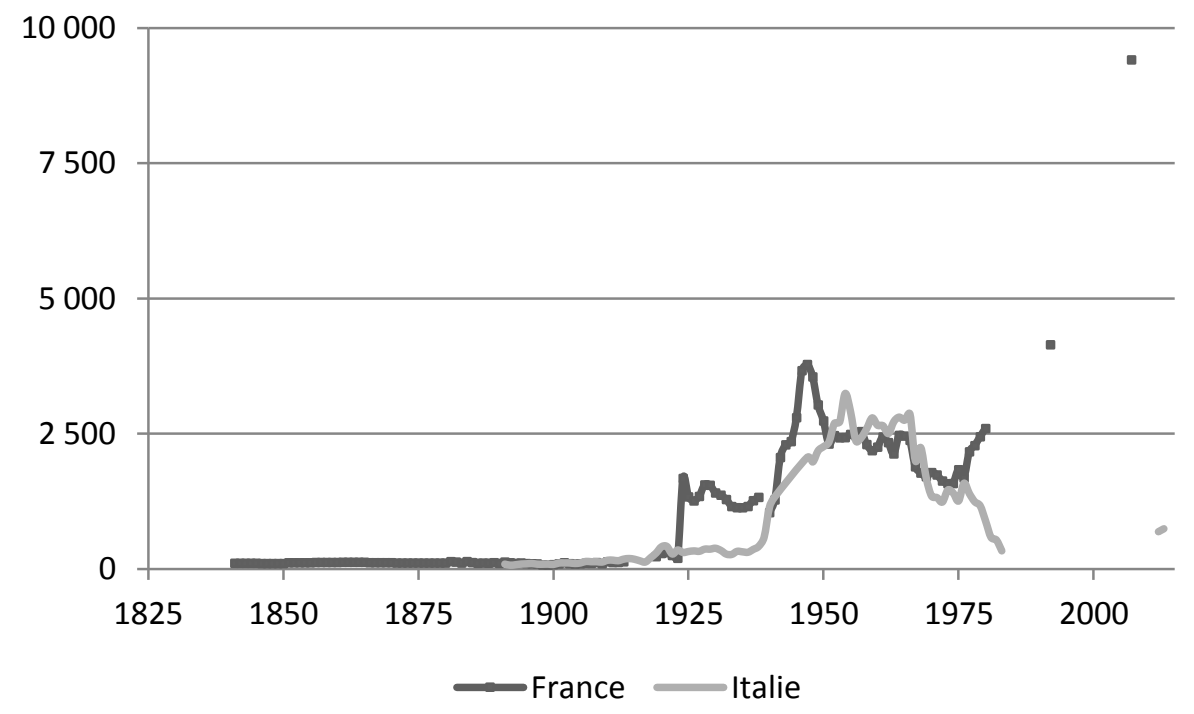

(a) Sources : Ministère de la Justice, Compte général; Belmokhtar, 1996 ; Belmokhtar, 2009 ; Istat, 2011 ; communication personnelle de la Direction générale de la statistique du Ministère de la Justice italien.

(b) Champs : en France, ce sont les adoptés simples dont le jugement d'adoption a été prononcé par un tribunal de première instance / grande instance, par année de jugement d'adoption (données annuelles de 1841 à 1980, et données ponctuelles en 1992 et 2007) ; en Italie, ce sont les adoptions simples (adozioni ordinarie), par année de jugement d'adoption (données annuelles de 1891 à 1983, données indisponibles depuis 1984).

Indépendamment des différences de calendrier de modification du droit de l'adoption d'un pays à l'autre, il est intéressant de noter que les évolutions du nombre annuel d'adoptés simples sont parallèles jusqu'au milieu des années 1970 : le nombre d'adoptés reste faible 
jusqu'au début du $\mathrm{XX}^{\mathrm{e}}$ siècle, puis il augmente fortement en France suite à la Première Guerre mondiale et en Italie suite à la Seconde Guerre mondiale, puis il connaît son maximum dans les deux pays suite à la Seconde Guerre, avant de baisser jusqu'au milieu des années 1970. En France comme en Italie, il semble que c'est la hausse du nombre de mineurs adoptables suite aux guerres mondiales (orphelins de guerre et enfants abandonnés) qui, de pair avec l'introduction d'une adoption simple de mineurs dans le droit, a fortement contribué à faire varier le nombre annuel d'adoptés simples jusqu'au milieu des années 1970. Même si l'on ne dispose pas de renseignements précis sur l'identité des adoptés et de leurs adoptants en Italie, notons qu'en France, de 1923 jusqu'au milieu des années 1970, l'adoption simple est typiquement un acte par lequel une tante ou une belle-mère, célibataire ou veuve et sans enfant, adopte sa nièce ou sa belle-fille, qu'elle a contribué à élever lorsqu'elle était encore mineure (orpheline de mère), pour lui transmettre son patrimoine (Fine, 2008).

Depuis le milieu des années 1970, alors que le nombre annuel d'adoptés simples explose en France, il chute en Italie, à tel point qu'Istat a cessé de collecter le nombre d'adoptés simples en Italie dès 1983 (Figure 1). Alors qu'en France on compte sur la période récente sans doute plus de 10000 adoptions simples par an (Belmokhtar, 2009), en Italie on en compte moins de 1000 (688 en 2012 et 743 en 2013, selon une communication personnelle de la Direction générale de la statistique du Ministère de la Justice italien). Certes, contrairement au droit français le droit italien n'autorise pas les adoptions simples (adozioni di persone maggiori d'età) qui portent sur des mineurs ; mais en France depuis au moins les années 1990, 85 \% des adoptions simples portent sur des majeurs, l'âge moyen des adoptés simples se situant autour de 33 ans (Belmokhtar, 1996 ; Belmokhtar, 2009). Et surtout, le droit italien permet depuis 1983 l'adoption simple intrafamiliale de mineurs (adozioni di casi particolari) ; mais de 1993 à 2012, le nombre d'adozioni di casi particolari est toujours resté inférieur à 1000 par an (minimum de 491 en 1993, maximum de 711 en 2008) (Giustizia Minorile, 2003, p. 15. Giustizia Minorile, 2014, p. 9). Par conséquent, le faible nombre d'adoptions simples en Italie n'est sans doute pas principalement dû aux spécificités du droit italien de l'adoption.

Alors, comment expliquer que les nombres d'adoptés simples sont aussi similaires jusqu'aux années 1970, mais divergent depuis lors ? En France, le nombre d'adoptés simples explose depuis le milieu des années 1970 en lien avec l'explosion des recompositions familiales après divorce (Mignot, 2015a). En effet, depuis les années 1970, la hausse des adoptions simples en France est due à la hausse des adoptions simples du bel-enfant par le beau-parent; en d'autres termes le beau-parent, le plus souvent sans enfant, adopte le belenfant qu'il a contribué à élever, afin de lui transmettre son héritage (Martial, 2000 ; Martial 2003). Par contraste, les recompositions familiales restent relativement rares en Italie, en raison de la relative rareté des divorces. Rappelons que depuis le milieu des années 1970 on compte de deux à sept fois moins de divorces par an en Italie qu'en France, et que l'indicateur conjoncturel de divortialité est de trois à huit fois moindre en Italie qu'en France (Ined, 2014). En revanche, le taux de remariage des divorcés semble inférieur en France à ce qu'il est en Italie, tout au moins sur la période récente $(2,1 \%$ des divorcés présents en France en 2012 se sont remariés cette année-là (Insee, 2014), contre $3 \%$ en Italie (Istat, 2013)). Quant à savoir si les familles recomposées qui ont le plus de chances de donner lieu à une adoption simple (c'est-à-dire les familles recomposées qui incluent non seulement un parent résidant avec son enfant mais aussi un conjoint sans enfant) sont proportionnellement plus nombreuses en France qu'en Italie, on l'ignore. Au total, c'est vraisemblablement la relative rareté des divorces et donc le faible nombre de familles recomposées qui explique que les adoptions simples sont beaucoup plus rares en Italie qu'en France. Cela n'implique pas, toutefois, qu'aucun autre mécanisme ne soit à l'œuvre. 
Abordons maintenant le nombre d'adoptés pléniers. L'adoption plénière existe en France depuis 1939 (d'abord sous le nom de « légitimation adoptive » de 1939 à 1966), et en Italie depuis 1967. Mais depuis que l'adoption plénière existe dans les deux pays, les nombres d'adoptés pléniers y sont de même ordre de grandeur, d'environ 2500 à 5000 par an (Figure 2). Si l'on observe une hausse du nombre annuel d'adoptés en France jusqu'à la fin des années 1970 (Houdaille et Nizard, 1977 ; Marmier-Champenois, 1978), et en Italie jusqu'à la fin des années 2000, les tendances sont désormais à la stagnation: le nombre annuel d'adoptés n'augmente plus, ni en France ni en Italie. Pour mieux comprendre ce que révèle ce plafonnement du nombre annuel d'adoptés pléniers en France et en Italie, il convient d'aborder les profils des adoptés pléniers.

Figure 2. Nombre annuel d'adoptés pléniers en France et en Italie, 1952-2008

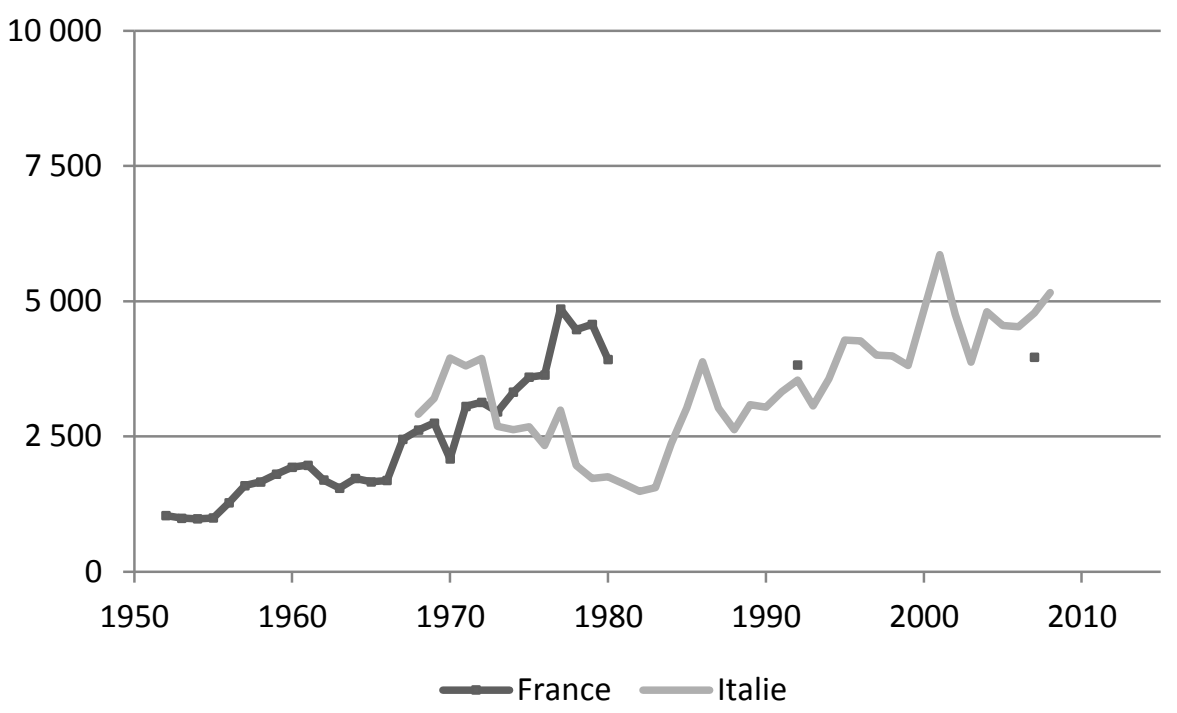

(a) Sources: Ministère de la Justice, Compte général; Belmokhtar, 1996 ; Belmokhtar, 2009 ; Istat, 2011.

(b) Champs : en France, ce sont les mineurs adoptés pléniers (bénéficiaires de «légitimation adoptive » de 1952 à 1966, et «adoptés pléniers » depuis) dont le jugement d'adoption a été prononcé par un tribunal de première instance / grande instance, par année de jugement d'adoption (données annuelles de 1952 à 1980, et données ponctuelles en 1992 et 2007) ; ces données ne tiennent donc pas compte des mineurs adoptés pléniers dont le jugement d'adoption a été prononcé à l'étranger (sur ce point, voir Figure 4); en Italie, ce sont les adoptions plénières (adozioni speciali de 1968 à 1983, et adozioni di minori depuis), par année de jugement d'adoption (données annuelles de 1968 à 2008).

\section{Le nombre et le profil des adoptés pléniers nationaux et internationaux}

Si aucune information n'est, à notre connaissance, publiée à propos des adoptés simples italiens (sauf que, comme les adoptés simples français, ils ont toujours été presque exclusivement des nationaux), il est possible de comparer les adoptés pléniers français et italiens, au moins sur la période récente. Dans quelle mesure les mouvements d'adoption plénière nationale et internationale sont-ils similaires en France et en Italie ? Et les adoptés pléniers français et italiens se ressemblent-ils ?

\section{a. Le faible nombre de mineurs nationaux adoptables plénièrement}

Depuis au moins les années 1990 le nombre de mineurs nationaux adoptables en adoption plénière est, en Italie comme en France, faible, mais il est stable en Italie et décroissant en France (Figure 3). En France, le nombre de mineurs nationaux adoptables (en stock) est passé de plus de 4000 au début des années 1990 à un peu plus de 2000 dans les années 2010. En Italie, le nombre de mineurs nationaux adoptables (toujours en stock) n'a jamais dépassé 2000 du milieu des années 1990 au milieu des années 2000. Au regard du nombre de candidats à l'adoption munis de l'agrément, environ trois à douze fois plus élevé 
que le nombre de mineurs adoptables (selon l'année et le pays), les mineurs adoptables sont insuffisamment nombreux (Observatoire national de l'enfance en danger, 2007-2013; Commissione per le adozioni internazionali, 2014). En France comme en Italie, la libéralisation de la contraception et de l'interruption volontaire de grossesse a réduit le nombre de naissances non désirées et, plus encore, le nombre d'abandons de nouveau-nés. Par exemple, le nombre d'accouchements sous le secret en France est passé d'environ 2000 par an à la fin des années 1960 à environ 1000 par an dans les années 1990 et 600 par an dans les années 2000 (Villeneuve-Gokalp, 2001). Parallèlement, le nombre d'enfants abandonnés (en flux) est d'environ 1000 par an dans les années 2000 (Observatoire national de l'enfance en danger, 2007-2013 ; Fréchon et al., 2009 ). C'est pourquoi le nombre de mineurs nationaux adoptables est à un niveau si bas, aussi bien en France et en Italie que dans les autres pays occidentaux (United Nations, 2009).

Figure 3. Nombre de mineurs nationaux adoptables plénièrement en France et en Italie, 1991-2012

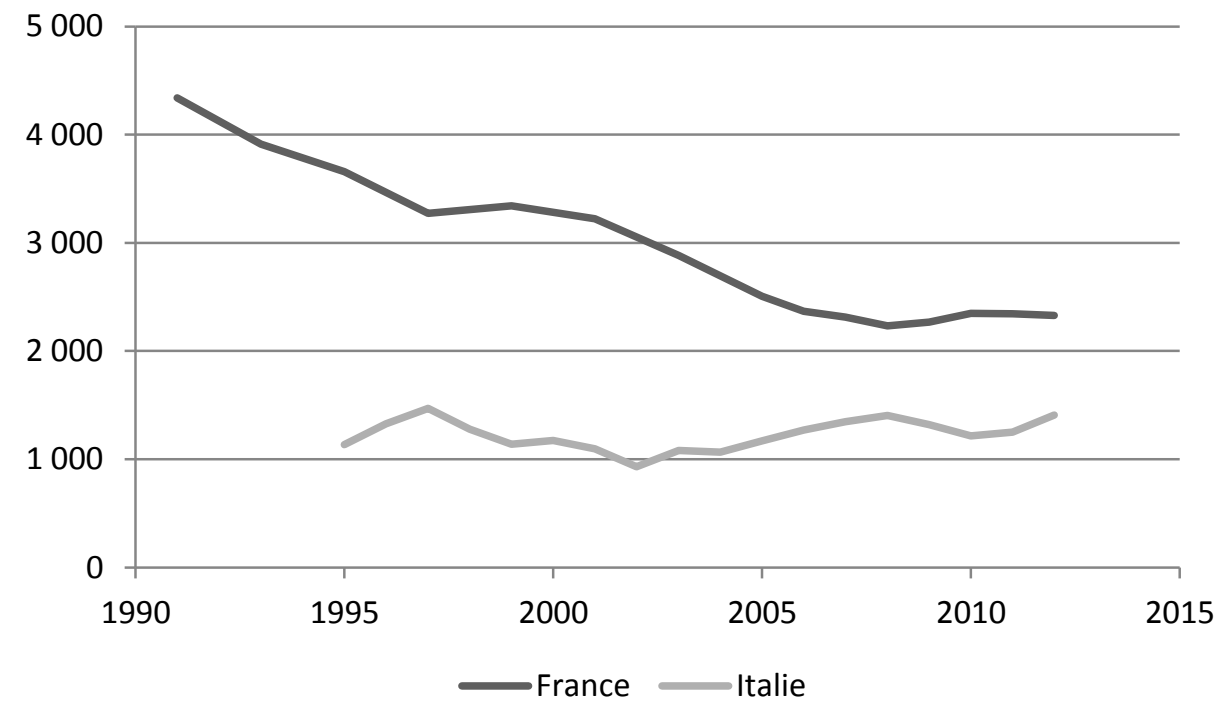

(a) Sources : Observatoire national de l'enfance en danger, 2007-2013; Istituto degli Innocenti, 2008. Giustizia Minorile, 2014.

(b) Champ: mineurs nationaux adoptables pléniers («pupilles de l'État » en France et minori in stato di adottabilità en Italie).

b. Le développement puis la récente baisse des adoptions internationales

Le faible nombre de mineurs nationaux adoptables et la longueur de la procédure d'adoption nationale ont conduit les candidats à l'adoption, d'abord en France puis aussi en Italie, à se tourner vers l'adoption internationale. Il en va de même dans la plupart des pays occidentaux, dans lesquels l'adoption internationale a été bien étudiée (Weil, 1984), notamment par le démographe britannique Peter Selman (Selman, 2002, 2006, 2010, 2012).

De 1990 à 2005 (en France) ou 2010 (en Italie), on compte ainsi autour de 3000 adoptés internationaux par an, la tendance temporelle étant plutôt à la hausse (Figure 4). Depuis 2011, toutefois, le nombre d'adoptés baisse, particulièrement en France, mais aussi en Italie. Sous l'influence de l'enrichissement et de la pacification des traditionnels pays d'origine des adoptés internationaux, mais aussi de la baisse de leur fécondité, du développement des politiques sociales et familiales d'aide à l'enfance, et de la baisse du stigmate lié aux naissances illégitimes, le nombre d'enfants de ces pays qui sont orphelins ou abandonnés baisse, tandis que le nombre d'adoptions par des nationaux croît. En outre, un nombre croissant de pays d'origine ont ratifié la Convention de La Haye, qui stipule qu'il est préférable, dans l'intérêt supérieur de l'enfant, que les enfants soient adoptés par des proches 
de leur famille ou, à défaut, par des nationaux, l'adoption ne devant être internationale qu'en dernier recours. Cette ratification a conduit de nombreux pays d'origine à restreindre drastiquement le nombre d'adoptions internationales, dans le but de prévenir le trafic d'enfants et plus généralement de promouvoir l'intérêt des mineurs. En conséquence, le faible nombre d'enfants adoptables en France et en Italie s'observe aussi, notamment depuis 2011, à l'international (Mignot, 2015b). La baisse du nombre de mineurs adoptables à l'international permet par exemple à la Chine d'exiger que les enfants chinois adoptables ne soient adoptés que par des couples hétérosexuels mariés, dont les deux membres ont le baccalauréat et travaillent, et ne souffrent pas d'obésité morbide. De même, au Brésil, le développement récent de l'adoption par les nationaux ne laisse plus à l'adoption internationale que des enfants «à besoins spécifiques », c'est-à-dire âgés, en fratrie et/ou malades ou handicapés.

Figure 4. Nombre de mineurs internationaux adoptés en France et en Italie, 1991-2014

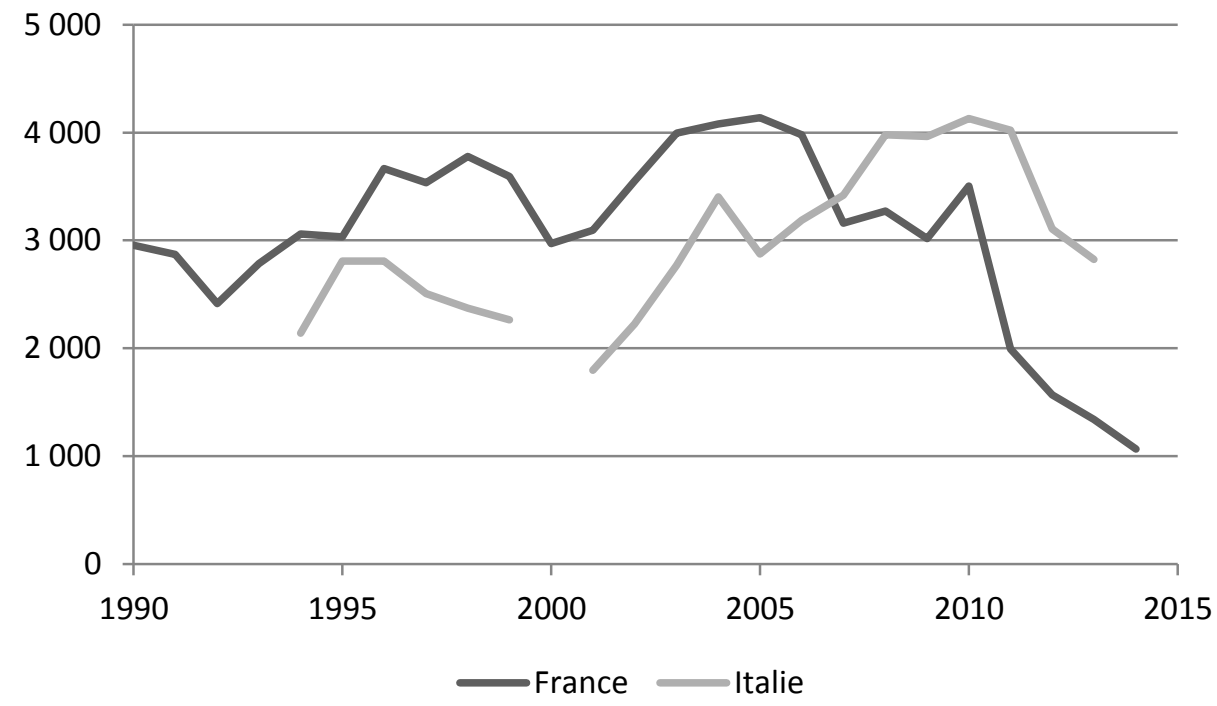

(a) Sources : Ministère des Affaires étrangères, 2014 ; Commissione per le adozioni internazionali, 2014.

(b) Champ : mineurs internationaux autorisés à entrer sur le territoire du pays de l'adoptant (dont la quasitotalité sont adoptés pléniers).

Les données rétrospectives de plus long terme qui sont disponibles (mais qui ne permettent pas de décrire les années les plus récentes) permettent de retracer la période depuis laquelle les adoptions internationales se sont peu à peu substituées aux adoptions nationales (Figure 5 ; sur la France voir Denéchère, 2011). Si jusqu'à la fin des années 1980 la part des adoptés internationaux était sans doute supérieure en France à ce qu'elle était en Italie, du début des années 1990 jusqu'au début des années 2010 environ les deux tiers des adoptés sont des adoptés internationaux, aussi bien en France qu'en Italie. Toutefois, depuis le début des années 2010, la baisse du nombre de mineurs internationaux adoptés (pléniers) en France et en Italie (observée sur la Figure 4, mais non représentée sur la Figure 5, qui repose sur des données différentes), de pair avec la stagnation du nombre de mineurs nationaux adoptables et, par suite, adoptés (pléniers) en France et en Italie (Figure 3), ont contribué à faire baisser récemment la part des adoptés pléniers annuels qui sont de nationalité étrangère. De nos jours, ce ne sont plus deux tiers, mais à peine la moitié des adoptés pléniers qui sont de nationalité étrangère. Globalement, les deux pays ont donc connu des trajectoires similaires, au moins depuis la fin des années 1980. Une raison majeure en est que les candidats à l'adoption français comme italiens sont confrontés non seulement à un faible nombre de mineurs nationaux adoptables, mais aussi au déclin du nombre de mineurs internationaux adoptables (Mignot, 2015b). 


\section{Figure 5. Part des adoptés pléniers annuels qui sont de nationalité étrangère, en France et en Italie, 1967-2008}

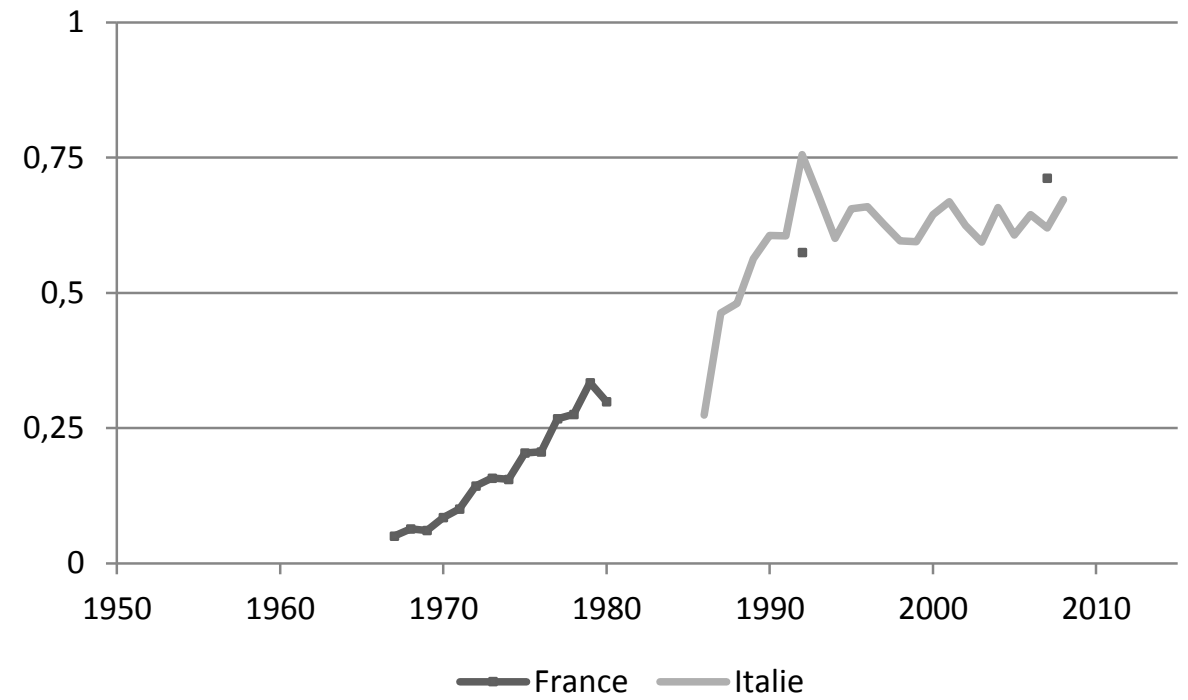

(a) Sources : Ministère de la Justice, Compte général; Belmokhtar, 1996 ; Belmokhtar, 2009 ; Istat, 2011.

(b) Champs : en France, ce sont les mineurs adoptés pléniers dont le jugement d'adoption a été prononcé par un tribunal de première instance / grande instance (et non pas directement transcrit sur les registres d'état civil par le parquet de Nantes), par année de jugement d'adoption (données annuelles de 1967 à 1980, et données ponctuelles en 1992 et 2007) ; en Italie, ce sont les adoptions plénières (adozioni speciali de 1968 à 1983, et adozioni di minori depuis), par année de jugement d'adoption (données annuelles de 1986 à 2008).

\section{c. Le profil des adoptés pléniers internationaux}

Depuis (au moins) les années 2000, les adoptants internationaux français et italiens ont des profils similaires : ce sont, pour la grande majorité, des couples mariés dont les membres ont aux alentours de 40 ans, qui sont plus fortement diplômés que la moyenne, et qui sont, sinon stériles, du moins inféconds. Parmi environ 350 familles italiennes (non représentatives) comprenant au moins un enfant de 7 à 11 ans, on a aussi montré que celles qui comprennent des adoptés internationaux ont d'autres caractéristiques distinctives: les parents adoptifs entretiennent, entre eux, une relation de meilleure qualité que la moyenne, ils sont mieux entourés, et ils éprouvent un degré de bien-être psychosocial supérieur à celui des parents non adoptifs (Rosnati et al., 2013). La différence majeure entre les adoptants internationaux français et italiens est qu'une partie des adoptants français sont des personnes agissant seules, tandis que pour des raisons juridiques tous les adoptants italiens sont des couples mariés. Comparons maintenant les profils des adoptés pléniers internationaux en France et en Italie depuis les années 2000, par sexe, âge et pays d'origine.

Alors que depuis le début des années 2000 les garçons représentent tout près de $50 \%$ des adoptés pléniers internationaux en France (Halifax et Villeneuve-Gokalp, 2005 ; Ministère des Affaires étrangères, 2014), ils représentent de $55 \%$ à $61 \%$ des adoptés pléniers internationaux en Italie (Istituto degli innocenti, 2008). Cette relative surreprésentation des garçons parmi les adoptés pléniers en Italie s'explique sans doute moins par les préférences des adoptants italiens - qui ne sont pas autorisés à formuler une préférence sur le sexe de l'adopté - que par la surreprésentation des garçons parmi les mineurs adoptables des pays dans lesquels ils tendent à adopter. Notamment, les adoptants italiens tendent à adopter en Russie ou en Ukraine, deux pays qui confient à l'adoption internationale surtout des garçons. Ainsi, parmi les mineurs adoptés en Russie de 2003 à 2013 par des Français, on compte de $60 \%$ à $66 \%$ de garçons (Halifax et Villeneuve-Gokalp, 2005 ; Ministère des Affaires étrangères, 2014). L'égale représentation des garçons et des filles parmi les adoptés en France s'explique donc par le fait que les adoptants français tendent non seulement à adopter dans 
des pays qui donnent à l'adoption internationale surtout des garçons, mais aussi dans des pays qui donnent à l'adoption internationale surtout des filles. Ainsi, parmi les mineurs adoptés en Chine et au Vietnam de 2003 à 2013 par des Français, on compte seulement de $2 \%$ à $47 \%$ de garçons (Halifax et Villeneuve-Gokalp, 2005 ; Ministère des Affaires étrangères, 2014).

Depuis le début des années 2000, les adoptés internationaux tendent à être de plus en plus âgés à l'adoption, aussi bien en France qu'en Italie. Par exemple, la part de mineurs internationaux adoptés qui ont moins de cinq ans à l'adoption est passée en France de $82 \%$ en 2004 à $67 \%$ en 2013, et en Italie elle est passée de $64 \%$ en 2000 à $47 \%$ en 2013. La principale raison de cette évolution est commune aux deux pays : en accord avec la Convention de La Haye, les pays d'origine des mineurs tendent à ne placer à l'adoption internationale que les mineurs qui sont les moins aisément adoptables par des nationaux, inclus les mineurs les plus âgés. Autrement dit, au fur et à mesure que l'adoption nationale se développe dans les traditionnels pays d'origine des adoptés internationaux, les seuls mineurs qui restent confiés à l'adoption internationale sont les mineurs relativement âgés.

Ajoutons que l'âge n'est pas la seule caractéristique individuelle qui rend un mineur plus difficilement adoptable : le fait qu'un mineur soit membre d'une fratrie inséparable, ou qu'il souffre d'une maladie ou d'un handicap physique ou mental, sont d'autres caractéristiques qui réduisent ses chances d'être adopté. Or, depuis la seconde moitié des années 2000, entre la moitié et les deux tiers des adoptés internationaux en France et en Italie sont des enfants «à besoins spécifiques », c'est-à-dire âgés ou en fratrie ou souffrant d'un handicap. Il est clair que de nos jours les candidats à l'adoption occidentaux sont en concurrence avec les candidats à l'adoption des pays d'origine des mineurs afin de se voir confier les mineurs les plus demandés, à savoir les nouveau-nés en bonne santé.

Enfin, depuis (au moins) les années 2000, les pays dans lesquels les candidats français et italiens vont adopter sont relativement différents (Selman, 2012). Alors que les Français adoptent en Asie (Chine, et Vietnam jusqu'à ce que ce pays signe la Convention de La Haye en 2011), en Amérique (Colombie, Haïti) et en Afrique (Ethiopie, Madagascar), et n'adoptent que depuis très récemment en Europe (Russie), les Italiens adoptent surtout en Europe (Russie, Ukraine, Bulgarie, Biélorussie, Pologne), et seulement secondairement en Amérique (Colombie, Brésil) ou en Afrique (Ethiopie).

Les raisons de cette différence ne sont pas évidentes. Tout d'abord, une part de la surreprésentation, en Italie, des adoptés d'origine russe pourrait s'expliquer par le fait que l'Italie a signé en 2008 un accord bilatéral sur l'adoption avec la Russie. Cela dit, une telle explication ne porte que sur la période postérieure à 2008. Ensuite, une part de la surreprésentation, en France, des adoptés originaires de pays francophones peut s'expliquer par le fait que les candidats à l'adoption internationale tendent à se diriger, quand c'est possible, vers des pays dont ils maitrisent la langue, en l'occurrence des pays tels le Vietnam, Haïti ou Madagascar. Plus généralement, la relative diversité des pays d'origine des adoptés internationaux en France peut être liée à l'importance de l'adoption dans la politique étrangère française, qui a permis à des organismes autorisés pour l'adoption de nouer des contacts avec un grand nombre de pays différents. Enfin, la relative rareté, en Italie, des adoptions d'enfants chinois peut s'expliquer par le fait que les candidats italiens à l'adoption ne peuvent pas adopter par démarche individuelle dans un pays de leur choix (ils ne peuvent adopter que dans un pays dans lequel est présent un organisme italien autorisé pour l'adoption), ce qui peut les empêcher d'adopter dans certains pays où les candidats français, eux, adoptent. En effet, la procédure italienne d'adoption internationale est telle qu'une fois que le couple a obtenu l'agrément il donne mandat à un organisme autorisé pour l'adoption (ente autorizzato) pour conduire la procédure à l'étranger, jusqu'à l'apparentement. Plus généralement, on pourrait imaginer que les candidats italiens valorisent plus fortement que les candidats français le fait d'adopter des enfants blancs, qui pourraient passer pour leurs propres enfants si ces adoptés 
n'étaient pas déjà relativement âgés lors de l'adoption. Cela dit, les données disponibles ne permettent pas de l'affirmer avec certitude (sur la construction de l'identité ethnique et nationale des adoptés internationaux en Italie, voir Ferrari et Rosnati, 2013 et Rosnati et Ferrari, 2014). Quoi qu'il en soit, il ne fait pas de doute que les différences de pays d'origine des adoptés internationaux en France et en Italie modifient le profil des adoptés : ce sont les pays dans lesquels les Italiens adoptent qui expliquent pourquoi ils adoptent relativement plus de garçons, et relativement plus de mineurs âgés, que les Français.

\section{Conclusion}

La comparaison des adoptions en France et en Italie depuis le $\mathrm{XIX}^{\mathrm{e}}$ siècle apporte plusieurs enseignements. Tout d'abord, la France et l'Italie ont connu des histoires du droit de l'adoption très proches, et aujourd'hui leurs droits de l'adoption simple et plénière sont fortement similaires. Si, dans ce contexte, on pouvait s'attendre à ce que le nombre d'adoptions simples suive des mouvements similaires dans les deux pays de la fin du XIX siècle jusqu'au milieu des années 1970, il est frappant que depuis lors le nombre d'adoptions simples reste très faible en Italie, alors qu'il explose en France. Cette divergence entre les pratiques d'adoption simple en France et en Italie révèle avant tout l'écart d'intensité du divorce qui s'est creusé entre les deux pays : si en France environ 10000 beaux-parents, pour la plupart sans enfants, adoptent chaque année leurs beaux-enfants afin de leur transmettre leur patrimoine, en Italie la rareté des recompositions familiales après divorce rend une telle pratique beaucoup plus rare.

Ensuite, les candidats français comme italiens à l'adoption plénière étant confrontés au faible nombre de mineur nationaux adoptables, ils ont été conduits, dès les années 1970 en France, puis aussi en Italie, à recourir à l'adoption plénière internationale. Depuis 2011, toutefois, le nombre d'adoptés internationaux baisse aussi bien en France qu'en Italie: diverses évolutions sociodémographiques, politiques et juridiques se mêlent pour réduire le nombre de mineurs adoptables à l'international. En conséquence du faible nombre de mineurs adoptables à l'international, les adoptés internationaux en France et en Italie tendent à être plus âgés, plus souvent en fratrie, et plus souvent malades ou handicapés qu'auparavant. On peut donc s'attendre à ce que les candidats à l'adoption, et notamment les couples stériles, cherchent à l'avenir à contourner le faible nombre de mineurs adoptables en ayant plus souvent recours aux diverses formes d'aide médicale à la procréation.

Il serait aussi intéressant de pouvoir comparer les profils des adoptés et des adoptants pléniers mais aussi des adoptés et des adoptants simples en Italie et en France sur le plus long terme. Mais reste à trouver les données italiennes analogues à celles du Compte général français. De telles analyses, répliquées sur d'autres pays comme l'Espagne ou l'Allemagne, permettraient certainement d'éclairer sous un nouveau jour l'histoire du droit et des pratiques de l'adoption dans les pays occidentaux, et plus généralement l'histoire démographique de ces pays. Enfin, restent à comparer les effets de l'adoption sur la santé et le bien-être des adoptés, puisque les études à ce jour disponibles sur ce point ont été réalisées de façon séparée - et selon des méthodologies qui ne sont pas directement comparables - en France (Halifax 2000 ; Halifax et Labasque 2013) et en Italie (Rosnati 2010 ; Chistolini 2010 ; Segatto et Dal Ben 2013).

\footnotetext{
${ }^{2}$ Je tiens à remercier Francesca Caroccia, Paola Ronfani et pour leurs précieux commentaires sur des versions préliminaires du présent article. Je remercie aussi Matteo Sgarzi pour son aide, ainsi que la Direction générale de la statistique du Ministère de la Justice italien pour avoir accepté de me communiquer ses données récentes sur l'adoption simple.
} 


\section{Références bibliographiques}

- BELMOKHTAR Zakia, 2009, «L'adoption simple et plénière en 2007 : des projets différents », Infostat Justice, 106, p. 1-6.

- BELMOKHTAR Zakia, 1996, «Les adoptions simples et plénières en 1992 », Infostat Justice, 46, p. 1-4.

- BRAND Elisabeth, 1985, «Adoption - Italie », Revue internationale de droit comparé, 37(3), p. 631-651.

- CARP E. Wayne, 1998, Family Matters: Secrecy and Disclosure in the History of Adoption, Cambridge, Harvard University Press, 304 p.

- CASELLI Graziella, VALLIN Jacques, WUNSCH Guillaume (dir.), 2001, Démographie. Analyse et synthèse I. la dynamique des populations, Paris, INED, 552p.

- CENTER FOR ADOPTION POLICY, 2014, « Overview of Italian Adoption Law ». Disponible à l'adresse : http://www.adoptionpolicy.org/pdf/eu-italy.pdf6.

- CHISTOLINI Marco, 2010, La famiglia adottiva. Come accompagnarla e sostenerla, Milan, FrancoAngeli, 256p.

- COMMISSIONE PER LE ADOZIONI INTERNAZIONALI, 2014, Dati e prospettive nelle adozioni internazionali. Rapporto sui fascicoli dal $1^{\circ}$ gennaio al 31 dicembre, Firenze, Istituto degli Innocenti, 115 p. Disponible à l'adresse : http://www.commissioneadozioni.it/media/143019/report statistico 2013.pdf.

- CONFÉRENCE DE LA HAYE DE DROIT INTERNATIONAL PRIVÉ, 2014, «Texte et signataires». Disponible à l'adresse : http://www.hcch.net/index_fr.php?act=conventions.status\&cid=69.

- DENÉCHÈRE Yves, 2011, Des enfants venus de loin. Histoire de l'adoption internationale en France, Paris, Colin, 408 p.

- DAVIS Mary Ann, 2013, «Demographics of Gay and Lesbian Adoption and Family Policies », in Baumle Amanda K. (dir.), International Handbook on the Demography of Sexuality, Dordrecht, Springer, p. 383-401.

- DAVIS Mary Ann, 2011, Children for Families or Families for Children. The Demography of Adoption Behavior in the U.S., Dordrecht, Springer, 220p.

- FERRARI Laura, ROSNATI Rosa, 2013, «Internationally Adopted Adolescents: How Do They Integrate Ethnic and National Identity? », Italian Journal of Sociology of Education, 5(3), p. 45-61.

- FINE Agnès, 2008, «Regard anthropologique et historique sur l'adoption. Des sociétés lointaines aux formes contemporaines », Informations sociales, 146(2), p. 8-19.

- FINE Agnès, 1998, Adoptions. Ethnologie des parentés choisies, Paris, Éditions de la Maison des sciences de l'homme, $311 \mathrm{p}$.

- FISHER Allen P., 2003, «Still "Not Quite As Good As Having Your Own"? Toward a Sociology of Adoption », Annual Review of Sociology, 29(1), p. 335-361.

- FRÉCHON Isabelle, GUYAVARCH Emmanuelle, HALIFAX Juliette, 2009, «État des lieux de l'enfance en danger, sources et données disponibles en France », Santé, société et solidarité, 1, p. 39-47.

- GIUSTIZIA MINORILE, 2014, Dati statistici relativi all'adozione anni 2000-2012, Rome, Giustizia Minorile, 13p. Disponible à l'adresse: http://www.giustiziaminorile.it/statistica/approfondimenti/Adozione_Serie_Storiche.pdf

- GIUSTIZIA MINORILE, 2003, L'applicazione della Legge 4 maggio 1983 n. 184 "Disciplina dell'adozione e dell'affidamento dei minori" negli anni 1993-1999. Analisi statistica, Rome, Giustizia Minorile, 33p. Disponible à l'adresse : http://www.giustiziaminorile.it/statistica/approfondimenti/Adozioni 1993 99.pdf.

- GUTTON Jean-Pierre, 1993, Histoire de l'adoption en France, Paris, Publisud, 194 p.

- HALIFAX Juliette, 2007, L'adoption plénière en France : de l'établissement d'une filiation légale à la constitution d'une filiation sociale, Thèse de démographie sous la direction de François Héran, Lille, Atelier national de reproduction des thèses, 513 p. 
- HALIFAX Juliette, 2000, «L'insertion sociale des enfants adoptés. Résultats de l'enquête "Adoption internationale et insertion sociale" », Dossiers et recherches de l'Ined, 98, p. 1-57.

- HALIFAX Juliette, VILLENEUVE-GOKALP Catherine, 2005, «L'adoption en France : qui sont les adoptés, qui sont les adoptants ? », Population et sociétés, 417, p. $1-4$.

- HALIFAX Juliette, LABASQUE Marie-Véronique, 2013, Étude relative au devenir des enfants adoptés en France et à l'international. Rapport final, Amiens : CREAI de Picardie, $130 \mathrm{p}$.

- HAMilton Laura, CHENG Simon, POWELL Brian, 2007, "Adoptive Parents, Adaptive Parents: Evaluating the Importance of Biological Ties for Parental Investment », American Sociological Review, 72(1), p. 95-116.

- HERMAN Ellen, 2008, Kinship by Design. A History of Adoption in the Modern United States, Chicago, University of Chicago Press, 368 p.

- HOUDAILLE Jacques, NIZARD Alfred, 1977, «L'adoption », Population et sociétés, 108, p. 1-4.

- INED, 2014, «Base de données Démographie des pays développés ». Disponible à l'adresse : http://www.ined.fr/fr/tout-savoir-population/chiffres/bases-donnees/donneespays-developpes/.

- INSEE, 2014, «Statistiques d'état civil sur les mariages en 2012 ». Disponible à l'adresse : http://www.insee.fr/fr/themes/detail.asp?ref id=ir-irsocsd20122.

- ISTAT, 2013, «Il matrimonio in Italia », Statistiche Report, p. 1-12.

- ISTAT, 2011, «Serie storiche. Tavola 6.9 Provvedimenti giudiziari in materia di stato delle persone - Anni 1891-2008 (valori assoluti). Adozioni di minori (di cui : adozioni di minori stranieri); Adozioni ordinarie ». Disponible à l'adresse : http://seriestoriche.istat.it/index.php?id=7\&user 100ind pi1\%5Bid pagina\%5D=49\&c Hash=66f9eb5b9dc25bcee546ed696f1da59a.

- ISTITUTO DEGLI INNOCENTI, 2008, Come cambia l'adozione internazionale in Italia. Le coppie e i bambini nel monitoraggio della Commissione per le adozioni internazionali negli anni 2000-2007, Firenze, Istituto degli Innocenti. Disponible à l'adresse :

http://www.commissioneadozioni.it/media/34762/411 come\%20cambia\%201 adozione .pdf.

- JABLONKA Ivan, 2006, Ni père ni mère. Histoire des enfants de l'Assistance publique (1874-1939), Paris, Seuil.

- LEFAUCHEUR Nadine, 2006, «De la tradition française au droit à la vérité de la biographie - ou du recours à l'histoire dans les débats parlementaires sur l'accouchement dit sous X », Clio. Histoire, femmes et sociétés, 24.

- LOVELOCK Kirsten, 2000, «Intercountry Adoption as a Migratory Practice: A Comparative Analysis of Intercountry Adoption and Immigration Policy and Practice in the United States, Canada and New Zealand in the Post W.W.II Period », International Migration Review, 34(3), p. 907-949.

- MARMIER-CHAMPENOIS Marie-Pierre, 1978, L'adoption. Effectivité de la loi du 11 juillet 1966. Approche des résultats de l'institution, Paris, Ministère de la Justice, $275 \mathrm{p}$.

- MARMIER Marie-Pierre, 1969, Sociologie de l'adoption. Etude de sociologie juridique, Paris, LGDJ, 422p.

- MARTIAL Agnès, 2003, S'apparenter. Ethnologie des liens de familles recomposées, Paris, Éditions de la Maison des sciences de l'homme, 309p.

- MARTIAL Agnès, 2000, «L'adoption de l'enfant du conjoint dans les familles recomposées », in Fine Agnès, Neirinck Claire (dir.), Parents de sang, parents adoptifs. Approches juridiques et anthropologiques de l'adoption. France, Europe, USA, Canada, Paris, LGDJ, p. 189-209. 
- MIGNOT Jean-François, 2015a, «L'adoption simple en France : le renouveau d'une institution ancienne (1804-2007) », Revue française de sociologie, 3, à paraître.

- MIGNOT Jean-François, 2015b, «L'adoption internationale dans le monde: les raisons du déclin », Population et sociétés, 519, p. 1-4.

- MINISTÈRE DE LA JUSTICE, 1980, Annuaire statistique de la Justice, Paris, Ministère de la Justice.

- MINISTÈRE DE LA JUSTICE, 1961-1976, Compte général de l'administration de la Justice criminelle et de la Justice civile et commerciale, Paris, Ministère de la Justice.

- MINISTÈRE DE LA JUSTICE, 1933-1960, Compte général de l'administration de la Justice civile et commerciale et de la Justice criminelle, Paris, Ministère de la Justice.

- MINISTÈRE DE LA JUSTICE, 1841-1932, Compte général de l'administration de la Justice civile et commerciale en France et en Algérie, Paris, Ministère de la Justice.

- MINISTÈRE DES AFFAIRES ÉTRANGÈRES, 2014, «Statistiques de 1'adoption internationale de l'Agence française de l'adoption». Disponible à l'adresse : http://www.agence-adoption.fr/le-paysage-de-ladoption-internationale/les-statistiques/, consulté en mai 2014.

- MURAT Pierre, 2008, «Les transformations de la famille. Quel impact sur les finalités de l'adoption ? », Informations sociales, 146(2), p. 20-33.

- MYKYTYN-GAZZIERO Olha, 2014, Enfants placés en Ukraine. De l'exclusion sociale à l'abandon d'enfant. Récits de mères, Paris, Karthala, 289p.

- NEIRINCK Claire, 2000, «L'évolution de 1'adoption », in Fine Agnès, Neirinck Claire (dir.), Parents de sang, parents adoptifs. Approches juridiques et anthropologiques de l'adoption. France, Europe, USA, Canada, Paris, LGDJ, p. 343361.

- OBSERVATOIRE NATIONAL DE L'ENFANCE EN DANGER, 2007-2013, La situation des pupilles de l'État au 31 décembre, Rapports 2007-2013.

- ROHRBASSER, Jean-Marc, 2014, « Hécatombe », in Rohrbasser Jean-Marc (dir.), Bouleversements démographiques de la Grande Guerre, Paris, INED, p. 9-18.

- ROSNATI Rosa, 2010, Il legame adottivo. Contributi internazionali per la ricerca e l'intervento, Milan, Unicopli, 275p.

- ROSNATI Rosa, FERRARI Laura, 2014, «Parental Cultural Socialization and Perception of Discrimination As Antecedents For Transracial Adoptees' Ethnic Identity », Procedia - Social and Behavioral Sciences, 140, p. 103-108.

- ROSNATI Rosa, RANIERI Sonia, BARNI Daniela, 2013, «Family and Social Relationships and psychosocial Well-Being in Italian Families With Internationally Adopted and Non-Adopted Children », Adoption Quarterly, 16, p. 1-16.

- SAVARD Nathalie, 2010, La théorie de l'attachement. Une approche conceptuelle au service de la protection de l'enfance, Paris, ONED.

- SEGATTO Barbara, DAL BEN Anna, 2013, «The Recovery Function of Adoption. A Study Carried Out in the Italy's Veneto Region », Interdisciplinary Journal of Family Studies, 18(2), p. 98-113.

- SELMAN Peter, 2012, «Global Trends in Intercountry Adoption: 2001-2010», Adoption Advocate, 44, p. 1-17.

- SELMAN Peter, 2010, «The Rise and Fall of Intercountry Adoption in the $21^{\text {st }}$ Century », International Social Work, 52(5), p. 575-594.

- SELMAN Peter, 2006, «Trends in Intercountry Adoption: Analysis of Data from 20 Receiving Countries, 1998-2004 », Journal of Population Research, 23(2), p. 183-204.

- SELMAN Peter, 2002, "Intercountry Adoption in the New Millennium; the "Quiet Migration" Revisited », Population Research and Policy Review, 21, p. 205-225.

- SÉNAT, 2014, «L'adoption». Disponible à l'adresse : http://www.senat.fr/lc/lc12/lc12_mono.html.

- UNITED NATIONS, 2009, Child Adoption: Trends and Policies, New York, United Nations Publications, $463 \mathrm{p}$. 
- VILLENEUVE-GOKALP Catherine, 2011, «Les femmes qui accouchent sous le secret en France, 2007-2009 », Population, 1, p. 135-169.

- VOISIN Joëlle, GEORGES Philippe, 2011, Audit du fonctionnement du conseil national d'accès aux origines personnelles (CNAOP). Rapport définitif, Paris, IGAS.

- WEIL R.H., 1984, «International Adoptions: The Quiet Migration », International Migration Review, 18, p. 276-293. 